\title{
Orchestration of B Lymphocyte Activation and Motility by the ERM Protein Ezrin
}

\author{
N. Parameswaran, S. Panigrahi, and N. Gupta
}

Department of Immunology, Cleveland Clinic, 9500 Euclid Avenue, NE40, Cleveland, OH 44195

Appropriate B cell responses hinge on finely balanced signal transduction pathways as well as regulated membrane remodeling [1]. Membrane remodeling requires its uncoupling from the underlying cortical cytoskeleton but its regulation and impact on B cell function is poorly understood [2]. Members of the ezrin-radixin-moesin (ERM) family act as linkers of the plasma membrane and the cortical actin cytoskeleton [3]. We have identified ezrin as a regulator of membrane-cytoskeletal uncoupling in response to both B cell antigen receptor (BCR) [4] and chemokine stimulation. Both stimuli induce dephosphorylation of ezrin on amino acid T567 in the actin-binding domain of ezrin, resulting in conformational inactivation of ezrin. Expression of a mutant of ezrin (T567D) that constitutively links the membrane to the actin cytoskeleton interferes with BCR-induced lipid raft coalescence, and SDF-1 $\alpha$-dependent B cell migration. Our data support a critical role for T567 dephosphorylation in the early membrane remodeling events associated with antigen and chemokine signaling. Mice with a conditional deletion of ezrin in the $\mathrm{B}$ cell lineage exhibit defects in peripheral B cell homeostasis. Ezrin-deficient B cells from these mice show impaired in vitro migration and in vivo homing. Our data suggest that ezrin has the potential to shape activation and migration associated with normal B cell homeostasis and immune response as well as lymphomagenesis and metastasis.

\section{References}

[1] N. Gupta, and A.L. DeFranco, Sem. Cell Dev. Biol. 18 (2007) 616.

[2] A. Viola, and N. Gupta, Nat. Rev. Immunol. 7 (2007) 889.

[3] A. Bretscher, Curr. Opin. Cell Biol. 11 (1999) 109.

[4] N. Gupta et al., Nat. Immunol. 7 (No 6) (2006) 625.

[5] This research was supported by grants from the NIH (DK068292), Cancer Research Institute and the Department of Defense. The help of Dr. Alex Huang of Case Western Reserve University is gratefully acknowledged. 